\title{
Escherichia Coli CRISPR/CAS System Evolved with Bacteriophage: A Case Report
}

\author{
Chi-Dung Yang ${ }^{1,2,4}$, Hsi-Yuan Huang ${ }^{3}$, Sirjana Shrestha ${ }^{1,4}$ and Hsien-Da Huang*1,4 \\ ${ }^{1}$ Institute of Bioinformatics and Systems Biology, National Chiao Tung University, Taiwan \\ ${ }^{2}$ Institute of Population Health Sciences, National Health Research Institutes, Taiwan \\ ${ }^{3}$ Department of Laboratory Medicine, China Medical University Hospital, Taiwan
}

${ }^{4}$ Department of Biological Science and Technology, National Chiao Tung University, Taiwan

Received: June 18, 2018; Published: June 25, 2018

*Corresponding author: Hsien-Da Huang, Professor of Bioinformatics, computational biology, and systems Biology College of Biological Science and Technology, National Chiao Tung University, Hsin-Chu, Taiwan

\begin{abstract}
Background: Clustered Regularly Interspaced Short Palindromic Repeats (CRISPR) is a part of an adaptive immune system in prokaryotes and occurring in diverse forms of bacteria.

Case Presentation: We presented the results of sequence analysis which can help in understanding the presence of CRISPR-mediated antiphage ability in both pathogenic and non-pathogenic strains of Escherichia coli.

Conclusion: Endogenous CRISPR spacers with imperfect matches are the candidates of anti-phage function in genetically distant strains of E. coli.
\end{abstract}

Keywords: CRISPR/Cas System; Bacteriophage; Escherichia Coli; Spacers

Abbreviations: CRISPR: Clustered Regularly Interspaced Short Palindromic Repeats; CAS: CRISPR-Associated Protein

\section{Background}

CRISPR is an acronym that stands for Clustered Regularly Interspaced Short Palindromic Repeats. It is an important part of an adaptive microbial immune system that occurs in wide varieties of bacteria [1]. CRISPR I and CRISPR II are two types of CRISPR elements that have been recognized in the Escherichia coli K-12 strain. CRISPR I is followed by eight cas genes namely, cas1, cas2, cas3, cas A, casB, casC, casD and case in CRISPR element I. The last five genes encode the proteins to form a Cascade like complexes. Two genes cas 1 and cas 2 are responsible for adding exogenous spacers in to CRISPR region which lies near the leader sequence [2]. However, CRISPR II element is not found to be surrounded by such cas genes [3]. The procurement of new spacers provide host with an immunological memory to distinguish an invaded DNA molecules. The functional mechanism of CRISPR/Cas system is a hierarchical process. The results from our previous study indicated that two endogenous spacers; CR1-7 and CR2-6, which are relatively conserved can hinder replication of P1 phage in E. coli K12 strain [4]. However, the comprehensive correlation between the CRISPR spac ers of pathogenic and non-pathogenic strains of E. coli and their phages still remains unclear.

\section{Case Presentation}

In this case study, we demonstrated the results of sequence analysis which can help understanding the presence of CRISPR-mediated anti-phage ability in pathogenic and non-pathogenic strains of E. coli. We collected E. coli spacers from CRISPRdb [5] and applied BLASTN program to compare the similarity between these spacers with the genome sequence of several Enterobacter phages, including P1, P2, T4, T5, T7, $\lambda$, $\phi X 147, \mathrm{MS} 2, \mathrm{Q} \beta$, and M13. The different spacers targeting the same phage were found in genetically distant strains (Supplementary File 1). Moreover, multiple spacers against the same phage were found in many strains, which was similar to the presence of multiple P1 specific spacers in E. coli K12 strain (Supplementary File 2). The results indicated that the CRISPR/Cas system in E. coli had actively acquired spacers from the phage which infected them rather than a random event that occurred when searching for base pairing between short sequences. 
Supplementary File 1:

\begin{tabular}{|c|c|c|c|c|c|c|c|c|}
\hline E. Coli Strains $\backslash$ Phages & P1 & P2 & Lambda & T4 & T7 & T5 & M13 & Qbeta \\
\hline UMN026 & 3 & & & 1 & & & & \\
\hline 42 & 2 & & & & & & & \\
\hline $\mathrm{P} 12 \mathrm{~b}$ & 3 & 1 & & & & & & \\
\hline ETECH10407 & 1 & & 1 & & & & & \\
\hline ED1a & 3 & & & & & & & \\
\hline 083:H1 str. NRG 857C & 1 & 2 & & & & & & \\
\hline LF82 & 1 & 1 & 1 & & & & & \\
\hline IAI1 & 2 & 1 & & & 1 & & & \\
\hline E24377A & 1 & & & 1 & & & & \\
\hline ATCC 8739 & 3 & 2 & & & & 1 & 1 & \\
\hline MG1655 & 5 & & 1 & 1 & 1 & & & \\
\hline BW2952 & 5 & & 1 & 1 & 1 & & & \\
\hline DH1 & 5 & & 1 & 1 & 1 & & & \\
\hline UMNK88 & 2 & 1 & & 1 & & & & \\
\hline 026:H11 11368 & 1 & 1 & 1 & & & & & 1 \\
\hline 0111:H- 11128 & 1 & 1 & 2 & & & & & \\
\hline ETEC H10407 & 1 & & & & & & & \\
\hline BL21(DE3) & 1 & 1 & & & & & & \\
\hline B REL606 & 1 & 1 & & & & & & \\
\hline UTI89 & & 2 & 1 & & & & & \\
\hline UM146 & & 1 & 1 & & & & & \\
\hline S88 & & 1 & 1 & & & & & \\
\hline IHE3034 & & 1 & 1 & & & & & \\
\hline 55989 & & 1 & 1 & 1 & & & & \\
\hline APEC 01 & & 1 & 1 & & & & & \\
\hline 0152:H28 SE11 & & 1 & & & & & & \\
\hline 0139:H28 E24377A & & 1 & & & & & & \\
\hline $09 \mathrm{HS}$ & & & 1 & & & & 1 & \\
\hline $\mathrm{w}$ & & & & 1 & & 1 & & \\
\hline K011FL & & & & 1 & & 1 & & \\
\hline SMS-3-5 & & & & & & & & 1 \\
\hline sum $=$ & 42 & 21 & 14 & 9 & 4 & 3 & 2 & 2 \\
\hline
\end{tabular}

Strain $=48$

Strain with CRISRP $=42$

Number of Spacer $=688$

Selected Phages: P1, P2, Lambda, T4, T7, T5, M13, Qbeta

The parameters used in BLAST are the same as a default setting, but only matches have base-pairing region spanning greater than 15 base-pairs were reported here. 
Supplementary File 2:

\begin{tabular}{|c|c|c|c|c|c|}
\hline Phage & E. coli strain & CRISPR ID & Spacer & Protospacer & \\
\hline \multicolumn{6}{|l|}{ P1 } \\
\hline & UMN026 & NC_011751_6 & GCTGGTGGCGCGGGCAAACGGAACAATCCCGC & $\begin{array}{l}\text { GCTGGTGGCGCGGGCGAACGGAA- } \\
\text { CAATCCCGC }\end{array}$ & \\
\hline & & NC_011751_6 & ACATGAATGTCGGTTCAGACCGTGTTTTTACC & $\begin{array}{l}\text { ACATGAATGTCGGCTGTGACCGTGT- } \\
\text { TTTCACC }\end{array}$ & A \\
\hline & & NC_011751_3 & TCGACGGGGTGCGGTAAAACCTTTGCGAACGC & $\begin{array}{l}\text { AGCTCTTGGTGCGGTAAAACCATTCAT- } \\
\text { TATCA }\end{array}$ & B \\
\hline & 042 & NC_017626_6 & CAAAGGACACCGGGAGGCACCCGGCACCGCA & $\begin{array}{c}\text { TAAAGGACACCGGGAAGCACCCGGCAC- } \\
\text { CGCA }\end{array}$ & \\
\hline & & NC_017626_6 & ACATGAATGTCGGTTCAGACCGTGTTTTTACC & $\begin{array}{l}\text { ACATGAATGTCGGCTGTGACCGTGT- } \\
\text { TTTCACC }\end{array}$ & A \\
\hline & $\mathrm{P} 12 \mathrm{~b}$ & NC_017663_8 & ACATGAATGTCGGTTCAGACCGTGTTTTTACC & $\begin{array}{l}\text { ACATGAATGTCGGCTGTGACCGTGT- } \\
\text { TTTCACC }\end{array}$ & A \\
\hline & & NC_017663_9_4 & TGGCTCTGCAACAGCAGCACCCATGACCACGTC & $\begin{array}{l}\text { CCGCTCTGCAACAAGAGCACAACGA- } \\
\text { CAACCGC }\end{array}$ & $\mathrm{F}$ \\
\hline & & NC_017663_9_1 & GACAGAACGGCCTCAGTAGTCTCGTCAGGCTCC & $\begin{array}{l}\text { CTTAACACAGGCTCAGGAAGCTCGT- } \\
\text { CAGGCTC }\end{array}$ & $\mathrm{E}$ \\
\hline & ETEC H10407 & NC_017633_7 & AAAATTTGTTGCAAAACTCGCTGTAAAAATAG & $\begin{array}{l}\text { TAATTTTGTTGCAAAACTCCCTACAAT- } \\
\text { CATGA }\end{array}$ & \\
\hline & ED1a & NC_011745_3 & TGCAAACGCTACTTGCTCATTGACCACGTAAG & $\begin{array}{l}\text { AAATTTCACCCCCAGCTTATTGACCACG- } \\
\text { TAAG }\end{array}$ & \\
\hline & & NC_011745_2 & GTGGCGCTAGCAATGAGATTTGCGGAAAAAAG & $\begin{array}{l}\text { ATTCGCGGCACACAGAGACTTGCG- } \\
\text { GAAAAAAG }\end{array}$ & \\
\hline & & NC_011745_2 & AGGAACCGCGTCAGCCCCCTGCAAAGCTCATT & $\begin{array}{l}\text { GAGCCGATCAATCTCCCCCTGCAAACGCT- } \\
\text { CATT }\end{array}$ & \\
\hline & $\begin{array}{l}\text { 083:H1 str. } \\
\text { NRG 857C }\end{array}$ & NC_017634_2 & AGCAGCTTTCCAGCGAGCGCGGTTAACTCACT & $\begin{array}{l}\text { GTCGCAAGACCAGCAAGCGCGGTTAAA- } \\
\text { GTTGC }\end{array}$ & $\mathrm{C}$ \\
\hline & LF82 & NC_011993_2 & AGCAGCTTTCCAGCGAGCGCGGTTAACTCACT & $\begin{array}{l}\text { GTCGCAAGACCAGCAAGCGCGGTTAAA- } \\
\text { GTTGC }\end{array}$ & $\mathrm{C}$ \\
\hline & IAI1 & NC_011741_4 & CCCGAAAGAGATTGCCAGCCAGCTTAATTTGC & $\begin{array}{l}\text { CGTCAGAAATAGTGCCATCCAGCT- } \\
\text { TAATTGCA }\end{array}$ & \\
\hline & & NC_011741_2 & CGACGGGGTGCGGTAAAACCTTTGCGAACGC & $\begin{array}{l}\text { TCAGCTCTTGGTGCGGTAAAACCAT- } \\
\text { TCATTAT }\end{array}$ & $\mathrm{B}$ \\
\hline & $\mathrm{E} 24377 \mathrm{~A}$ & NC_009801_1 & CGACGGGGTGCGGTAAAACCTTTGCGAACGC & $\begin{array}{l}\text { TCAGCTCTTGGTGCGGTAAAACCAT- } \\
\text { TCATTAT }\end{array}$ & B \\
\hline & ATCC 8739 & NC_010468_2 & CACGGCTGGCCATTTGAAATACCTGTTGCTCT & $\begin{array}{l}\text { CCGCATCCCCCATTTGAAATCCCTGTG- } \\
\text { GAGCA }\end{array}$ & $\mathrm{D}$ \\
\hline & & NC_010468_2 & CACGGCTGGCCATTTGAAATACCTGTTGCTCT & $\begin{array}{l}\text { CCGCATCCCCCATTTGAAATCCCTGTG- } \\
\text { GAGCA }\end{array}$ & $\mathrm{D}$ \\
\hline & & NC_010468_2 & GACAGAACGGCCTCAGTAGTCTCGTCAGGCTCC & $\begin{array}{l}\text { CTTAACACAGGCTCAGGAAGCTCGT- } \\
\text { CAGGCTC }\end{array}$ & $\mathrm{E}$ \\
\hline & $\begin{array}{l}\text { MG1655/ } \\
\text { BW25113 }\end{array}$ & NC_000913_5 & TGGCTCTGCAACAGCAGCACCCATGACCACGTC & $\begin{array}{l}\text { CCGCTCTGCAACAAGAGCACAACGA- } \\
\text { CAACCGC }\end{array}$ & $\mathrm{F}$ \\
\hline & & NC_000913_5 & GACAGAACGGCCTCAGTAGTCTCGTCAGGCTCC & $\begin{array}{l}\text { CTTAACACAGGCTCAGGAAGCTCGT- } \\
\text { CAGGCTC }\end{array}$ & $\mathrm{E}$ \\
\hline & & NC_000913_4 & GTAGTCCATCATTCCACCTATGTCTGAACTCC & $\begin{array}{l}\text { CATATCCCTCATTCCACCTACACTGAT- } \\
\text { TACCC }\end{array}$ & \\
\hline & & NC_000913_4 & TCAACATTATCAATTACAACCGACAGGGAGCC & $\begin{array}{l}\text { GCAACGTTAATGATTACAACCGAGCTATT- } \\
\text { AGC }\end{array}$ & \\
\hline & & NC_000913_4 & AAGCTGGCTGGCAATCTCTTTCGGGGTGAGTC & $\begin{array}{c}\text { AAGCTGGCTGGAATCACTCATCGAAA- } \\
\text { GTCAT }\end{array}$ & \\
\hline
\end{tabular}




\begin{tabular}{|c|c|c|c|c|c|}
\hline & BW2952 & NC_012759_5 & TGGCTCTGCAACAGCAGCACCCATGACCACGTC & $\begin{array}{l}\text { CCGCTCTGCAACAAGAGCACAACGA- } \\
\text { CAACCGC }\end{array}$ & $\mathrm{F}$ \\
\hline & & NC_012759_5 & GACAGAACGGCCTCAGTAGTCTCGTCAGGCTCC & $\begin{array}{l}\text { CTTAACACAGGCTCAGGAAGCTCGT- } \\
\text { CAGGCTC }\end{array}$ & $\mathrm{E}$ \\
\hline & & NC_012759_4 & GTAGTCCATCATTCCACCTATGTCTGAACTCC & $\begin{array}{l}\text { CATATCCCTCATTCCACCTACACTGAT- } \\
\text { TACCC }\end{array}$ & \\
\hline & & NC_012759_4 & TCAACATTATCAATTACAACCGACAGGGAGCC & $\begin{array}{l}\text { GCAACGTTAATGATTACAACCGAGCTATT- } \\
\text { AGC }\end{array}$ & \\
\hline & & NC_012759_4 & AAGCTGGCTGGCAATCTCTTTCGGGGTGAGTC & $\begin{array}{l}\text { AAGCTGGCTGGAATCACTCATCGAAA- } \\
\text { GTCAT }\end{array}$ & \\
\hline & DH1 & NC_017638_5 & TGGCTCTGCAACAGCAGCACCCATGACCACGTC & $\begin{array}{l}\text { CCGCTCTGCAACAAGAGCACAACGA- } \\
\text { CAACCGC }\end{array}$ & $\mathrm{F}$ \\
\hline & & NC_017638_5 & GACAGAACGGCCTCAGTAGTCTCGTCAGGCTCC & $\begin{array}{l}\text { CTTAACACAGGCTCAGGAAGCTCGT- } \\
\text { CAGGCTC }\end{array}$ & $\mathrm{E}$ \\
\hline & & NC_017638_4 & GTAGTCCATCATTCCACCTATGTCTGAACTCC & $\begin{array}{l}\text { CATATCCCTCATTCCACCTACACTGAT- } \\
\text { TACCC }\end{array}$ & \\
\hline & & NC_017638_4 & TCAACATTATCAATTACAACCGACAGGGAGCC & $\begin{array}{l}\text { GCAACGTTAATGATTACAACCGAGCTATT- } \\
\text { AGC }\end{array}$ & \\
\hline & & NC_017638_4 & AAGCTGGCTGGCAATCTCTTTCGGGGTGAGTC & $\begin{array}{l}\text { AAGCTGGCTGGAATCACTCATCGAAA- } \\
\text { GTCAT }\end{array}$ & \\
\hline & UMNK88 & NC_017641_5_4 & TGGCTCTGCAACAGCAGCACCCATGACCACGTC & $\begin{array}{l}\text { CCGCTCTGCAACAAGAGCACAACGA- } \\
\text { CAACCGC }\end{array}$ & $\mathrm{F}$ \\
\hline & & NC_017641_5_1 & GACAGAACGGCCTCAGTAGTCTCGTCAGGCTCC & $\begin{array}{l}\text { CTTAACACAGGCTCAGGAAGCTCGT- } \\
\text { CAGGCTC }\end{array}$ & $\mathrm{E}$ \\
\hline & $\begin{array}{c}\mathrm{O} 26: \mathrm{H} 11 \\
11368\end{array}$ & NC_013361_2_2 & GACAGAACGGCCTCAGTAGTCTCGTCAGGCTCC & $\begin{array}{l}\text { CTTAACACAGGCTCAGGAAGCTCGT- } \\
\text { CAGGCTC }\end{array}$ & $\mathrm{E}$ \\
\hline & 0111:H- 11128 & NC_013364_2_1 & GACAGAACGGCCTCAGTAGTCTCGTCAGGCTCC & $\begin{array}{l}\text { CTTAACACAGGCTCAGGAAGCTCGT- } \\
\text { CAGGCTC }\end{array}$ & $\mathrm{E}$ \\
\hline & ETEC H10407 & NC_017633_7_1 & GACAGAACGGCCTCAGTAGTCTCGTCAGGCTCC & $\begin{array}{l}\text { CTTAACACAGGCTCAGGAAGCTCGT- } \\
\text { CAGGCTC }\end{array}$ & $\mathrm{E}$ \\
\hline & BL21(DE3) & NC_012892_5_1 & GACAGAACGGCCTCAGTAGTCTCGTCAGGCTCC & $\begin{array}{l}\text { CTTAACACAGGCTCAGGAAGCTCGT- } \\
\text { CAGGCTC }\end{array}$ & $\mathrm{E}$ \\
\hline & B REL606 & NC_012967_5_1 & GACAGAACGGCCTCAGTAGTCTCGTCAGGCTCC & $\begin{array}{l}\text { CTTAACACAGGCTCAGGAAGCTCGT- } \\
\text { CAGGCTC }\end{array}$ & $\mathrm{E}$ \\
\hline \multicolumn{6}{|c|}{ P2 } \\
\hline & ATCC 8739 & NC_010468_3 & CATCCGGCGCTGAACATCGCCACCTGCCTAAC & $\begin{array}{l}\text { TGACAACGACTGAACATCGCCGCCTGCG- } \\
\text { GGCG }\end{array}$ & \\
\hline & & NC_010468_3 & CGACGTTTTCTAATATCACCCAGCAATCAATT & $\begin{array}{l}\text { TGCCACTTTCTCAATATCACCCAG- } \\
\text { CGACCCGAC }\end{array}$ & \\
\hline & & NC_010468_2 & TTCTTGCGGGTGTTGCAAATATTCTTCACGTA & $\begin{array}{l}\text { CGGCGGCGGGGGTTCCGAGATATTCT- } \\
\text { TCACATG }\end{array}$ & \\
\hline & UTI89 & NC_007946_3 & GCGCGCAGTGCCTGATAATCAATTTTGCTCAT & $\begin{array}{l}\text { AGGGGACGCGCCGGATAATCAATTTTCT- } \\
\text { TACC }\end{array}$ & \\
\hline & & NC_007946_3 & ATGAGCAAAATTGATTATCAGGCACTGCGCGC & $\begin{array}{l}\text { GGTAAGAAAATTGATTATCCGGCG- } \\
\text { CGTCCCCT }\end{array}$ & \\
\hline & UMNK88 & NC_017641_4 & AATTGATTGCTGGGTGATATTAGAAAACGTCG & $\begin{array}{l}\text { GTCGGGTCGCTGGGTGATATTGAGAAA- } \\
\text { GTGGCA }\end{array}$ & \\
\hline & UM146 & NC_017632_3 & ATGAGCAAAATTGATTATCAGGCACTGCGCGC & $\begin{array}{l}\text { GGTAAGAAAATTGATTATCCGGCG- } \\
\text { CGTCCCCT }\end{array}$ & G \\
\hline & S88 & NC_011742_3 & ATGAGCAAAATTGATTATCAGGCACTGCGCGC & $\begin{array}{l}\text { GGTAAGAAAATTGATTATCCGGCG- } \\
\text { CGTCCCCT }\end{array}$ & G \\
\hline & LF82 & NC_011993_3 & ATGAGCAAAATTGATTATCAGGCACTGCGCGC & $\begin{array}{l}\text { GGTAAGAAAATTGATTATCCGGCG- } \\
\text { CGTCCCCT }\end{array}$ & G \\
\hline & & NC_011993_3 & GCGCACGGCGTGGCGACAGAGAGCACGCCCGC & $\begin{array}{l}\text { GCGGACGGCCAGTGGCGA CAGATTGT- } \\
\text { CACCATTG }\end{array}$ & \\
\hline
\end{tabular}




\begin{tabular}{|c|c|c|c|c|c|}
\hline & IHE3034 & NC_017628_3 & ATGAGCAAAATTGATTATCAGGCACTGCGCGC & $\begin{array}{l}\text { GGTAAGAAAATTGATTATCCGGCG- } \\
\text { CGTCCCCT }\end{array}$ & G \\
\hline & IAI1 & NC_011741_4 & TTTGCCGCTGTCAGCATTGCTGGCGCGTAATA & $\begin{array}{c}\text { AATGCCGGTGTCAGCATTGAAGAAAC- } \\
\text { CGCCGC }\end{array}$ & \\
\hline & BL21(DE3) & NC_012892_4 & AGCTGGGCGAAATTTTGATTCATCGTGATGAC & $\begin{array}{c}\text { AACTGGGGAAATTTTGATTCCCGCTAT- } \\
\text { GTGA }\end{array}$ & $\mathrm{H}$ \\
\hline & B REL606 & NC_012967_4 & AGCTGGGCGAAATTTTGATTCATCGTGATGAC & $\begin{array}{l}\text { AACTGGGGAAATTTTGATTCCCGCTAT- } \\
\text { GTGA }\end{array}$ & $\mathrm{H}$ \\
\hline & 55989 & NC_011748_4 & TACCGGTGTGAATTACAGCACCACCGCCACCC & $\begin{array}{l}\text { ATGGGATACGGTCACCAGTACCACCGC- } \\
\text { CACCG }\end{array}$ & \\
\hline & APEC 01 & NC_008563_3 & ATGAGCAAAATTGATTATCAGGCACTGCGCGC & $\begin{array}{l}\text { GGTAAGAAAATTGATTATCCGGCG- } \\
\text { CGTCCCCT }\end{array}$ & G \\
\hline & $\begin{array}{l}\text { O152:H28 } \\
\text { SE11 }\end{array}$ & NC_011415_4 & AATAACTCGCGTAAATGCTCTGCGGCGCTACG & $\begin{array}{c}\text { TCAGGAGATAGTAAATGCTCTGCTGCT- } \\
\text { GTAAA }\end{array}$ & \\
\hline & $\mathrm{P} 12 \mathrm{~b}$ & NC_017663_8 & AGCCGGGCGAAATTTTGATTCATCGTGATGAC & $\begin{array}{l}\text { CAACTGGGGAAATTTTGATTCCCGCTAT- } \\
\text { GTGA }\end{array}$ & \\
\hline & $\begin{array}{l}\text { 083:H1 str. } \\
\text { NRG 857C }\end{array}$ & NC_017634_3 & TCATTCTGTACAGAAAGATCAACCATAATATT & $\begin{array}{l}\text { TGATTCTGTACAGGAAGACGCACGT- } \\
\text { TAGCTAT }\end{array}$ & \\
\hline & & NC_017634_3 & GCGCACGGCGTGGCGACAGAGAGCACGCCCGC & $\begin{array}{l}\text { GCGGACGGCCAGTGGCGACAGATTGT- } \\
\text { CACCATTG }\end{array}$ & \\
\hline & $\begin{array}{c}\mathrm{O} 26: \mathrm{H} 11 \\
11368\end{array}$ & NC_013361_2 & TACGTGAAGAATATTTGCAACACCCGCAAGAA & $\begin{array}{l}\text { CATGTGAAGAATATCTCGGAACCCCCGC- } \\
\text { CGCCG }\end{array}$ & $\mathrm{H}$ \\
\hline & 0111:H- 11128 & NC_013364_2 & TACGTGAAGAATATTTGCAACACCCGCAAGAA & $\begin{array}{l}\text { CATGTGAAGAATATCTCGGAACCCCCGC- } \\
\text { CGCCG }\end{array}$ & $\mathrm{H}$ \\
\hline & $\begin{array}{l}\text { O139:H28 } \\
\text { E24377A }\end{array}$ & NC_009801_3_15 & TTCAGGGGAGTTCGCAAGACCAGCGGAATCGG & $\begin{array}{l}\text { ATTGAGGGATTCCGCAAGACCAGTGCA- } \\
\text { CAGCT }\end{array}$ & \\
\hline \multicolumn{6}{|l|}{ lambda } \\
\hline & UTI89 & NC_007946_3_4 & GCGCGCAGTGCCTGATAATCAATTTTGCTCAT & $\begin{array}{l}\text { TCACGCAGTGCCTGAGAGTTAAT- } \\
\text { TTCGCTCAC }\end{array}$ & I \\
\hline & UM146 & NC_017632_3_2 & GCGCGCAGTGCCTGATAATCAATTTTGCTCAT & $\begin{array}{l}\text { TCACGCAGTGCCTGAGAGTTAAT- } \\
\text { TTCGCTCAC }\end{array}$ & I \\
\hline & S88 & NC_011742_3_5 & GCGCGCAGTGCCTGATAATCAATTTTGCTCAT & $\begin{array}{l}\text { TCACGCAGTGCCTGAGAGTTAAT- } \\
\text { TTCGCTCAC }\end{array}$ & I \\
\hline & LF82 & NC_011993_3_15 & GCGCGCAGTGCCTGATAATCAATTTTGCTCAT & $\begin{array}{l}\text { TCACGCAGTGCCTGAGAGTTAAT- } \\
\text { TTCGCTCAC }\end{array}$ & I \\
\hline & IHE3034 & NC_017628_3_5 & GCGCGCAGTGCCTGATAATCAATTTTGCTCAT & $\begin{array}{l}\text { TCACGCAGTGCCTGAGAGTTAAT- } \\
\text { TTCGCTCAC }\end{array}$ & I \\
\hline & APEC 01 & NC_008563_3_5 & GCGCGCAGTGCCTGATAATCAATTTTGCTCAT & $\begin{array}{l}\text { TCACGCAGTGCCTGAGAGTTAAT- } \\
\text { TTCGCTCAC }\end{array}$ & I \\
\hline & $\begin{array}{c}\text { 026:H11 } \\
11368\end{array}$ & NC_013361_2_8 & CTGCCGGGTGAAACCACTCGCGGCAGATCTTG & $\begin{array}{l}\text { ATGCAGGGTGAAACCATTCCCGGCAT- } \\
\text { TCATCG }\end{array}$ & $\mathrm{J}$ \\
\hline & 0111:H- 11128 & NC_013364_2_6 & CTGCCGGGTGAAACCACTCGCGGCAGATCTTG & $\begin{array}{l}\text { ATGCAGGGTGAAACCATTCCCGGCAT- } \\
\text { TCATCG }\end{array}$ & $\mathrm{J}$ \\
\hline & & NC_013364_2_3 & TCCAACCTTCCATGAGATACGCGCATTAGCGG & $\begin{array}{l}\text { TAAAACCTTCCATGTGATACGAGGGCG- } \\
\text { CGTAG }\end{array}$ & \\
\hline & $09 \mathrm{HS}$ & NC_009800_6_17 & TCAGGGATTTTTAATTGATGATATGCAGATAC & $\begin{array}{l}\text { TTCATCACTTTTAATTGATG- } \\
\text { TATATGCTCTCTT }\end{array}$ & \\
\hline & ETEC H10407 & NC_017633_7_5 & TCGCGCCAGCACGTCGTCCGGAATCATGATTC & $\begin{array}{c}\text { CGCCGCCAGCACGTCCGCCGGA- } \\
\text { CAGGCTGCAT }\end{array}$ & \\
\hline & MG1655 & NC_000913_4_11 & AGCGTGTTCGGCATCACCTTTGGCTTCGGCTG & $\begin{array}{l}\text { CCCATCTCTCCGCATCACCTTTGGTAAA- } \\
\text { GGTTC }\end{array}$ & $\mathrm{K}$ \\
\hline & BW2952 & NC_012759_4_11 & AGCGTGTTCGGCATCACCTTTGGCTTCGGCTG & $\begin{array}{l}\text { CCCATCTCTCCGCATCACCTTTGGTAAA- } \\
\text { GGTTC }\end{array}$ & K \\
\hline
\end{tabular}




\begin{tabular}{|c|c|c|c|c|c|}
\hline & DH1 & NC_017638_4_11 & AGCGTGTTCGGCATCACCTTTGGCTTCGGCTG & $\begin{array}{l}\text { CCCATCTCTCCGCATCACCTTTGGTAAA- } \\
\text { GGTTC }\end{array}$ & K \\
\hline \multicolumn{6}{|l|}{$\mathrm{T} 4$} \\
\hline & $\mathrm{W}$ & NC_017635_3_13 & TAAAGTAGAATAAAAATATTCGCATAACAGAC & $\begin{array}{l}\text { ATCTTTAGAATAAAAATATTCATCAAGA- } \\
\text { TATC }\end{array}$ & \\
\hline & UMNK88 & NC_017641_5_7 & TTTGCCACCCGAGTCCATAAATTCTTGATATGC & $\begin{array}{c}\text { CACTCGCGCTTCGGCAATAAATTCTTGA- } \\
\text { TATGT }\end{array}$ & \\
\hline & UMN026 & NC_011751_7_1 & ACAAATGATGCGCCAAAACCAAGACTTTTACA & $\begin{array}{l}\text { ACAAATGATGCGCCAATAATTTCAATA- } \\
\text { GTTTT }\end{array}$ & \\
\hline & K011FL & NC_016902_3_2 & GTCTGTTATGCGAATATTTTTATTCTACTTTA & $\begin{array}{l}\text { GATATCTTGATGAATATTTTTATTCTAAA- } \\
\text { GAT }\end{array}$ & \\
\hline & E24377A & NC_009801_3_7 & CTCAAAAAACTTGAAATCAAAACCGGTGAAGA & $\begin{array}{l}\text { ATTCAAAAACTTGAATCAAAACCAAT- } \\
\text { GAATA }\end{array}$ & \\
\hline & 55989 & NC_011748_4_10 & ATTAAAGGATTATTTTGATGAGTCTGAAAAAT & $\begin{array}{l}\text { GTTAAAGCATTATTTTGATCTAC- } \\
\text { TATAAAAGA }\end{array}$ & \\
\hline & MG1655 & NC_000913_4_10 & TCAACATTATCAATTACAACCGACAGGGAGCC & $\begin{array}{c}\text { ACAACATTATCACTTAAAAATTTAAAAT- } \\
\text { TACT }\end{array}$ & $\mathrm{N}$ \\
\hline & BW2952 & NC_012759_4_10 & TCAACATTATCAATTACAACCGACAGGGAGCC & $\begin{array}{c}\text { ACAACATTATCACTTAAAAATTTAAAAT- } \\
\text { TACT }\end{array}$ & $\mathrm{N}$ \\
\hline & DH1 & NC_017638_4_10 & TCAACATTATCAATTACAACCGACAGGGAGCC & $\begin{array}{c}\text { ACAACATTATCACTTAAAAATTTAAAAT- } \\
\text { TACT }\end{array}$ & $\mathrm{N}$ \\
\hline \multicolumn{6}{|l|}{$\mathrm{T} 5$} \\
\hline & W & NC_017635_3_13 & TAAAGTAGAATAAAAATATTCGCATAACAGAC & $\begin{array}{l}\text { TAAAGGAGAATAAAAATGATCCG- } \\
\text { CAACGTTTCT }\end{array}$ & \\
\hline & K011FL & NC_016902_3_2 & GTCTGTTATGCGAATATTTTTATTCTACTTTA & $\begin{array}{l}\text { AGAAACGTTGCGGATCATTTTTAT- } \\
\text { TCTCCTTTA }\end{array}$ & \\
\hline & ATCC 8739 & NC_010468_2_20 & CTCGATCAGGAAAATGAATTCCTGGAAAAAAA & $\begin{array}{l}\text { CATTTACAGGAAAATGATATTCCTGACG- } \\
\text { TATGG }\end{array}$ & \\
\hline \multicolumn{6}{|l|}{$\mathrm{T} 7$} \\
\hline & IAI1 & NC_011741_3_8 & TAAACCACCAGCCAGACCACCAATTACCACAC & $\begin{array}{l}\text { GAAACCACCAGCGAGACCCATAGAGGT- } \\
\text { GATGA }\end{array}$ & 0 \\
\hline & MG1655 & NC_000913_4_6 & AAGCTGGCTGGCAATCTCTTTCGGGGTGAGTC & $\begin{array}{l}\text { GCGCTGGCTGGCATCTCTCCGATGTTC- } \\
\text { CAAC }\end{array}$ & 0 \\
\hline & BW2952 & NC_012759_4_6 & AAGCTGGCTGGCAATCTCTTTCGGGGTGAGTC & $\begin{array}{l}\text { GCGCTGGCTGGCATCTCTCCGATGTTC- } \\
\text { CAAC }\end{array}$ & 0 \\
\hline & DH1 & NC_017638_4_6 & AAGCTGGCTGGCAATCTCTTTCGGGGTGAGTC & $\begin{array}{l}\text { GCGCTGGCTGGCATCTCTCCGATGTTC- } \\
\text { CAAC }\end{array}$ & \\
\hline \multicolumn{6}{|l|}{ M13 } \\
\hline & $09 \mathrm{HS}$ & NC_009800_6_16 & GAGAGACGAAGCATGAAAAAATTAATAATATC & $\begin{array}{l}\text { GAGATTTTCAACATGAAAAAATTATTAT- } \\
\text { TCGC }\end{array}$ & \\
\hline & ATCC 8739 & NC_010468_2_15 & ATTACGCCGCCTCGCGTTTTTAGTCATTTCTA & $\begin{array}{c}\text { GCTAAAACGCCTCGCGTTCTTAGAATAC- } \\
\text { CGGA }\end{array}$ & \\
\hline \multicolumn{6}{|l|}{ Qbeta } \\
\hline & SMS-3-5 & NC_010498_3_13 & TCATACTGCTCCCACACCGAAAGCGCGGCAGC & $\begin{array}{l}\text { AGGAACTGCACCCGCTTCTGAAAGCG- } \\
\text { CGGCAAC }\end{array}$ & \\
\hline & $\begin{array}{l}\text { 026:H11 } \\
11368\end{array}$ & NC_013361_2_5 & AATCGTGTGTAAATTCGCGGCGGCTCCACTGG & $\begin{array}{c}\text { TAACTACCTGTCCCTGGCGGCGGCTC- } \\
\text { CAGTGG }\end{array}$ & \\
\hline
\end{tabular}


This idea is further supported with by the fact that E. coli 42 , UMN026, and P12b display almost nearly ideal matched spacers against P1 phage. We also found that several strains carried the same anti-P1 phage spacer as that of E. coli K12. E. coli DH1 and BW2952 strain showed the same CRISPR sequence as those of K12 strains. A spacer found in E. coli IAI1 was similar to the seventh spacer in CRISPR I locus of K12 (25/25, 100\% identical), but it can form long base-pairing region to P1 genome. Similarly, the last spacer in CRISPR II region was widespread in 11 strains (Supplementary File 2). The abundance of CRISPR spacers is shown in Figure 1. These spacers could pair with 16 nucleotides of phage sequence, which is as half the size of an E. coli type spacer. The spacers against P1 were the most abundant. On the contrary, only few spacers matched the genomes of M13 and Q $\beta$ with a statistically significant number of base pair. The general trend for all $E$. coli derived-CRISPR spacers was similar to that observed in the CRISPR region of E. coli K12 BW25113, which was used to conduct most of the experiments in the present case.

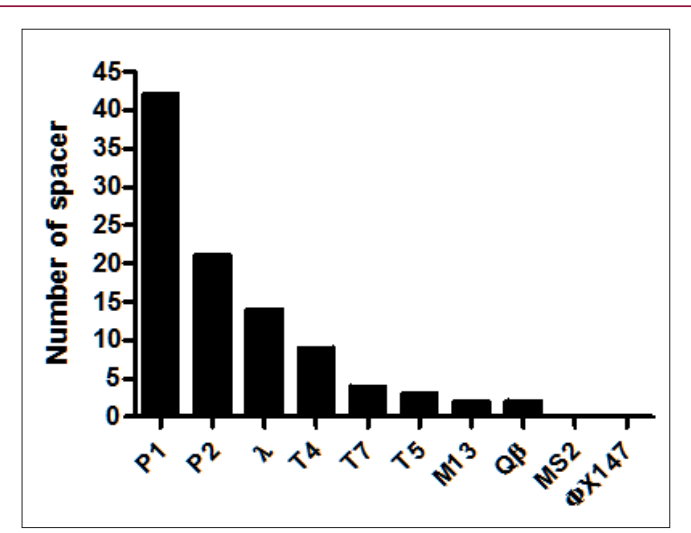

Figure 1: Distribution of antiphage spacers in E.coli in CRISPR database [5].

\section{Discussion}

Formerly it was disclosed that the high interrelationship between multilocus sequence typing tree and spacer repertoire was an indication that the CRISPR system in various strains of $E$. coli has lost immune function [6]. However, our results not only demonstrated that several endogenous CRISPR spacers in E. coli K12 strains still provide anti-phage function but also found the distribution of potential anti-phage spacers is more diverse than the ge- netic distance indicated by a phylogenic tree[7]. Therefore, the lack of significant immune effect in prior studies might be due to the accruement of plentiful of escape mutations in bacteriophage they studied $[2,8]$. Provided that phages with escape mutations might be used for replication, the rivalry between phage and bacteria establishes a pressure of natural selection to both species, which is an essential compelling force for phage diversification. In addition, a CRISPR deletion E. coli protein expression strain can be used as competent cell because the plasmids are highly stable in these mutant strains due to less interference on the replication and expression of protein expression plasmids.

\section{Conclusion}

The results of this case report revealed that endogenous spacer with flawed matches as the candidates of anti-phage function in genetically distant strains of E. coli. Furthermore, endogenous spacers with imperfect matches showing anti-phage function can be validated using spacer deletion strains.

\section{References}

1. Horvath P, Barrangou R (2010) CRISPR/Cas, the immune system of bacteria and archaea. Science 327(5962): 167-170.

2. Yosef I, Goren MG, Qimron U (2012) Proteins and DNA elements essential for the CRISPR adaptation process in Escherichia coli. Nucleic acids research 40(2): 5569-5576.

3. Pougach K, Semenova E, Bogdanova E, Datsenko KA, Djordjevic M, et al. (2010) Transcription, processing and function of CRISPR cassettes in Escherichia coli. Molecular microbiology 77(6): 1367-1379.

4. Yang CD, Chen YH, Huang HY, Huang HD, Tseng CP (2014) CRP represses the CRISPR/Cas system in Escherichia coli: evidence that endogenous CRISPR spacers impede phage P1 replication. Mol Microbiol 92(5): 1072-1091.

5. Grissa I, Vergnaud G, Pourcel C (2007) The CRISPRdb database and tools to display CRISPRs and to generate dictionaries of spacers and repeats. BMC bioinformatics 8: 172 .

6. Touchon M, Charpentier S, Clermont O, Rocha EP, Denamur E, et al. (2011) CRISPR distribution within the Escherichia coli species is not suggestive of immunity-associated diversifying selection. Journal of bacteriology 193(10): 2460-2467.

7. Lukjancenko O, Wassenaar TM, Ussery DW (2010) Comparison of 61 sequenced Escherichia coli genomes. Microbial ecology 60(4): 708-720.

8. Poranen MM, Ravantti JJ, Grahn AM, Gupta R, Auvinen P, et al. (2006) Global changes in cellular gene expression during bacteriophage PRD1 infection. J Virol 80(16): 8081-8088.
This work is licensed under Creative Commons Attribution 4.0 License

Submission Link: https://biomedres.us/submit-manuscript.php



